Comité de Expertos Enfermeros en Cuidados de UPP y Heridas de la Federación Argentina de Enfermería (CEECUPPYH-FAE)*

* Sandra Etchenique, Alberto Gugliara Cance, Ángel Aznar, Hernán Lascano, Walter Arce, Leonardo Benavídez, Miguel Martínez y Lorena Juárez

Coordinadores del estudio:

Sandra Etchenique ${ }^{1, *}$ y Maximiliano Saiz ${ }^{2}$

1. Licenciada en Enfermería. Diplomada en Cicatrización en Heridas por la Universidad Abierta Interamericana. Coordinadora General del CEECUPPYH-FAE.

Experta acreditada por el GNEAUPP. Enfermera Referente en Heridas por el Hospital de Clínicas José de San Martín (UBA). Directora de la Diplomatura para Enfermería en cuidados avanzados y cicatrización de heridas.

2. Consultor Independiente en Tecnologías de la Información. Especialista en IT. Experto en Seguridad Informática. Experto en redes acreditado por Microsoft. Colaborador del CEECUPPYH-FAE.

${ }^{*}$ Autor para correspondencia.

Correo electrónico: Comiteexpertos@yahoo.com.ar

Recibido el 5 de marzo de 2019; aceptado el 19 de julio de 2019

\section{Primer estudio nacional de prevalencia de úlceras por presión en Argentina, 2018. El primer paso para la maratón nacional de UPP}

\section{National marathon: the first step. 1st National Prevalence Study of UPP in Argentina 2018}

\section{RESUMEN}

Introducción: Las úlceras por presión (UPP) constituyen un importante problema de salud en todos los sistemas de salud. Es por ello por lo que el Comité de Expertos Enfermeros en Cuidados de UPP y Heridas de la Federación Argentina de Enfermería (CEECUPPYH-

FAE) decidió iniciar una estrategia nacional específica acerca del problema de las UPP, "La Maratón Nacional: El primer paso". En el presente artículo presentamos los datos correspondientes al primer estudio nacional de prevalencia de UPP en la República Argentina como punto de partida de nuestra maratón nacional. Metodología: Para ello se planteó un estudio nacional con los siguientes objetivos: obtener indicadores epidemiológicos sobre UPP en instituciones de salud de la República Argentina; identificar el riesgo de padecer UPP; determinar las características demográficas y clínicas de los pacientes prevalentes; determinar las características de las lesiones identificadas, e identificar medidas utilizadas en prevención de UPP. Para ello se realizó un estudio descriptivo transversal mediante encuesta online realizada a profesionales de enfermería entre el 1 de junio y el 30 julio de 2018. Se utilizaron las directrices de clasificación de EPUAP-NPUAP y los indicadores del GNEAUPP. Resultados: Participaron 200 profesionales de enfermería que relevaron 3755 encuestas en 22 de las 24 provincias del país. La prevalencia bruta de UPP fue del $22,55 \%$ en pacientes adultos, un 48,3\% de los pacientes habían desarrollado UPP nosocomiales; el 57\% de la muestra tenía edad para jubilarse (+ 60 ańos) y el 70\% pertenecía a servicios generales. Las localizaciones de las lesiones más frecuentes fueron sacro, talones y trocánteres, respectivamente. La prevalencia de

UPP en pacientes pediátricos fue del 8,35\%; un 69,44\% de los pacientes pediátricos presentaban UPP nosocomiales. El 76\% de los pacientes pediátricos estaban internados en cuidados generales y el 66\% presentaba riesgo bajo de padecer UPP. Las localizaciones más frecuentes fueron cabeza, sacro y talones. La prevalencia bruta de

UPP en neoonatos fue del 4,15\%, todos ellos presentaban UPP nosocomiales. El 66\% de los neonatos presentaba riesgo de padecer UPP y las localizaciones más frecuentes fueron cabeza y sacro.

Conclusiones: "La Maratón Nacional: El primer paso" es el primer estudio de investigación a nivel nacional sobre UPP en el que participaron profesionales de enfermería de todo el país y ha permitido obtener cifras de prevalencia a nivel nacional, así como de tendencias de

\section{ABSTRACT}

Introduction: Pressure ulcers (PU) are a major health problem in all Health Systems. That is why the Committee of Experts in UPP and Wound Nurses of the Argentine Nursing Federation (CEECUPPYH-FAE) decided to initiate a specific national strategy on the UPP problem, "The National Marathon: The First Step". In the present article we present the data corresponding to the first national study of PUs prevalence in the Argentine Republic as the starting point of our national marathon. Methodology: For this purpose, a national study was proposed with the following objectives: to obtain Epidemiological Indicators on PUs in Health Institutions of the Argentine Republic, to identify the risk of suffering PU, to determine the demographic and clinical characteristics of the patients of the prevalent patients, to determine the characteristics of identified lesions and to identify measures used in PU's prevention.A cross-sectional descriptive study was conducted through an online survey of Nursing Professionals between June 1 and July 30, 2018, using EPUAP-NPUAP classification guidelines and GNEAUPP indicators. Results: There were 200 nurses who surveyed 3755 surveys in 22 of the country's 24 provinces. The Gross Prevalence of PUs was $22.55 \%$ in adult patients, of which $48.3 \%$ of prevalent patients had developed nosocomial PUs. $57 \%$ of the sample is old enough to retire (+ 60 years), $70 \%$ belongs to general services. The most frequent lesion locations were sacrum, heels and trochanteres respectively. The prevalence of PUs in paediatric patients was $8.35 \% ; 69.4 \%$ of paediatric prevalent patients presented nosocomial PUs. $76 \%$ of the prevalent pediatric patients were hospitalized in general care and $66 \%$ had a low risk of UPP. The most frequent locations were head, sacrum and heels. The gross prevalence of PUs in neoonates was 4.15\%, all of them presenting nosocomial PUs. Sixty-six percent of neonates were at risk of $\mathrm{PU}$ and the most frequent locations were head and sacrum. Conclusions: "The National Marathon: The first step" is the First National Argentinian Research Study on PUs where nurses from all over the country participated and has allowed to obtain national prevalence figures, as well as 
prevención, lo que sin lugar a dudas es una información de gran utilidad para establecer estrategias nacionales para la mejora del problema de las UPP y monitorizar dicho problema en la Argentina.

PALABRAS CLAVE: Úlceras por presión, prevalencia, Argentina, prevención, riesgo. prevention trends, which without a doubt is a very useful information to establish national strategies for the improvement of the PUs problem and to monitor this problem in Argentina.

KEYWORDS: Pressure Ulcers, prevalence, Argentina, prevention, risk.

\section{У INTRODUCCIÓN}

Nadie pone en duda que las úlceras por presión (UPP) son, hoy en día, un importante problema de salud pública a nivel internacional ${ }^{1,2}$.

Las UPP producen elevados costos para el sistema de salud, y si bien existen medidas de prevención, continúan siendo un problema global que afecta tanto a los recursos humanos como financieros, pese a que se sabe que un alto porcentaje de las mismas se pueden prevenir ${ }^{3}$.

La Organización para la Cooperación y Desarrollo Económico (OCDE) realizó un documento de trabajo sobre salud en $2017^{4}$ donde afirmaba que las UPP ocupan el 14 . $^{\circ}$ lugar de morbilidad a nivel global y representan el $15 \%$ de los gastos en la actividad total de los hospitales. Hauck K et al. ${ }^{5}$ publicaron una investigación donde afirmaban que, si se comparan los años de vida sana perdidos a causa de los 6 eventos adversos declarados, las UPP representan el mayor valor con 13780 días de vida perdidos y 1127 días/cama perdidos en hospitales.

Las UPP generan dańos graves a la integridad de las personas, y pese a que son un indicador de calidad inherente a la atención brindada por el personal de salud, en Argentina hay escasos datos epidemiológicos y estos no se tienen en cuenta en el check list de las organizaciones más reconocidas que acreditan la calidad de atención y aspectos de seguridad del paciente.

Por este motivo, el Comité de Expertos Enfermeros en Cuidados de UPP y Heridas de la Federación Argentina de Enfermería (CEECUPPYH-FAE) decidió iniciar una estrategia nacional específica acerca del problema de las UPP, "La Maratón Nacional: El primer paso". Con ella, por primera vez en la historia, la enfermería argentina une sus esfuerzos para destapar la llamada "Epidemia bajo las sabanas", siguiendo el ejemplo del Grupo Nacional para el Estudio y Asesoramiento en Úlceras por Presión y Heridas Crónicas español (GNEAUPP) con el convencimiento de que la obtención, análisis y difusión de datos epidemiológicos nacionales argentinos relacionados con las UPP constituyen una herramienta básica para dimensionar este problema. También para el quiebre de una concepción cultural que las coloca como proceso inevitable y secundario de una persona que transita un proceso de enfermedad en una institución de salud.

Cuando el CEECUPPYH realizó el Primer Congreso sobre Cicatrización de Heridas, bajo el lema "Con las manos de todo el país: Úlceras ni una más”, ya se estaba proyectando su primer Estudio de Investigación como Comité Científico; han pasado 2 años y bajo el lema "Maratón Nacional: El primer paso" presenta el Primer Estudio de Prevalencia de UPP a nivel nacional.

En todas partes hay excepciones, pero aun así hemos encontrado muy buena predisposición por parte de colegas de todo el país. Un gran ejemplo fue la provincia de Chaco, donde por medio de una Resolución Ministerial, la Jefatura Provincial de Enfermería coordinó la recolección de datos junto a otras colegas referentes de la provincia; también se realizó una tarea exitosa en Córdoba, Santa Fe, Santiago del Estero y Entre Ríos. En otras no hubo la misma respuesta a nivel institucional global e incluso de muchos comités de ética; podemos deducir que los motivos van por dos vías: la primera es que en nuestro país los estudios de Enfermería tienen una orientación netamente asistencialista, no se fomenta la investigación durante la carrera de pregrado y, en consecuencia, no estamos familiarizados con los aspectos de aprobación de protocolos de investigación por parte de los diferentes comités, hecho concreto que vuelve muy "engorroso" el pedir las autorizaciones correspondientes. Por otro lado, las instituciones de salud todavía siguen viendo las UPP como un problema secundario que se debe mantener en secreto. De hecho, se esperaba mayor apoyo y colaboración de organizaciones abocadas a la seguridad del paciente y acreditadoras de calidad de atención.

Para finalizar esta introducción, el Comité quiere reforzar el concepto de que el conocimiento epidemiológico constituye la piedra angular para el diseño de políticas sanitarias de prevención como primera línea de defensa. Por este motivo, el CEECUPPYH-FAE, junto a colegas de todo el país, asumió la responsabilidad y compromiso de aunar fuerzas en esta difícil tarea dentro de la estrategia:

Maratón Nacional: El primer paso... para decir iiiúlceras ni una más!!!

Para ello se planteó, como objetivo general, obtener información epidemiológica sobre las UPP en instituciones de salud de la República Argentina, y como objetivos específicos:

- Establecer la prevalencia de pacientes con UPP internados en instituciones de salud públicas y privadas del territorio argentino.

- Identificar el riesgo de padecer UPP que tienen los pacientes internados en instituciones de salud públicas y privadas del territorio argentino.

- Determinar características demográficas y clínicas de los pacientes prevalentes de UPP.

- Determinar las características de las UPP identificadas en los pacientes prevalentes de UPP.

- Identificar los aspectos más relevantes de las políticas de las instituciones en relación con las UPP

\section{Y MÉTODO}

De acuerdo con los objetivos planteados para esta investigación, se disenó un estudio descriptivo observacional transversal (corte de prevalencia) en el que se estudiaron las características de la muestra seleccionada, identificando la ocurrencia del fenómeno y describiendo las variables que lo conforman en un universo definido por los pacientes internados en las instituciones de salud de la República Argentina.

Como criterio de inclusión se consideró a los pacientes con al menos 24 horas de internación en áreas generales, cirugía o áreas de cuidados intensivos en instituciones de salud ${ }^{7}$. Se excluyó a los pacientes con menos de 24 horas de internación y que no estuviesen ingresados en las áreas mencionadas.

Las variables de estudio fueron seleccionadas teniendo como referencia los datos mínimos establecidos por el European Pressure Ulcer 
Tabla 1. Características de los estudios seleccionados para el análisis

\begin{tabular}{l} 
Variables \\
Ubicación geográfica \\
de la institución sanitaria \\
Existencia de referente/grupo/comité de \\
UPP/cuidados de la piel o similar a nivel \\
institucional \\
\hline La institución realizó estudios de \\
investigación en relación con la \\
prevalencia de UPP \\
La institución cuenta con protocolos \\
escritos y vigentes para la prevención \\
y tratamiento de las UPP8 \\
\hline En el último año, la institución realizó \\
alguna capacitación en relación con \\
prevención y tratamiento de las UPP \\
\hline En el hospital se utiliza alguna escala \\
validada para evaluar riesgo de padecer UPP \\
\hline Grupo de insumos que utiliza la institución \\
habitualmente para prevenir UPP
\end{tabular}

Denominación formal por la institución de referente/grupo/etc. a quien se pueda consultar Estudios de prevalencia de UPP realizados por la institución

Protocolos escritos y puestos en marcha a nivel institucional

Capacitación brindada por la institución en estudio sobre prevención y tratamiento de UPP

Escala validada con la que enfermería evalúa el riesgo que tienen los pacientes de padecer UPP

Grupo de insumos que se utiliza a nivel institucional para prevenir UPP

$\begin{array}{ll}\text { Sala de internación } & \text { Lugar físico donde se encuentra internado } \\ \text { el paciente en el momento del estudio }\end{array}$

Edad del paciente

Sexo
Patología de ingreso
Riesgo de desarrollar UPP evaluado por
escala validada

Posee UPP al ingreso

El paciente posee actualmente UPP

Estadio y localización de UPP por paciente $^{12}$

\section{Género que posee el paciente} corresponda a neonatos, pediatría 0 adultos

En el momento del estudio el paciente posee lesión localizada en la piel y/o el tejido subyacente, por lo general sobre una ya ingresó con dicha lesión

\section{En el momento del estudio posee lesión} por lo general sobre una prominencia ósea, combinación con la cizalla
Diagnóstico médico por el que está internado

La valoración del riesgo para desarrollar UPP en el momento en que se toman los datos, medido a través de la aplicación de la EVRUPP según prominencia ósea, como resultado de la presión, o la presión en combinación con la cizalla, pero localizada en la piel y/o el tejido subyacente, como resultado de la presión, o la presión en

Clasificación de la úlcera por presión de acuerdo con Sacro, talones, isquiones, codos, trocánter, cabeza, orejas, acromion, el alcance en profundidad de la lesión. Se utilizará la omóplatos, escápulas, espalda, maléolos categorización establecida por la EPUAP (2009)

Femenino/masculino

Infecciosa, quirúrgica, clínica, oncológica

\section{Escala NSRAS ${ }^{10}$ : (neo): con riesgo/sin riesgo \\ Escala Braden Q11: (pediatría): bajo/medio/alto \\ Escala Braden': (adultos): bajo/medio/alto}

\section{Sí/no}

Sí/no

\section{Categorías}

Salta, Jujuy, Tucumán, Catamarca, Santiago del Estero, Formosa, Chaco, Corrientes, Misiones, Entre Ríos, La Rioja, Mendoza, San Juan y San Luis, Buenos Aires, Córdoba, La Pampa, Santa Fe, Chubut, Neuquén, Río negro, Santa Cruz, Tierra del Fuego, Antártida e Islas del Atlántico Sur

Sí/no

Sí/no

Sí/no

Sí/no

Sí/no

SEMP: sobrecolchones, almohadones, almohadas, cuñas 0 algún otro dispositivo que ayude a la redistribución de la presión Tópicos: cremas c/s, vitamina A, lociones, aceites esenciales, AGHO, silicona en espray, sulfadiazina de plata

Protección local: apósitos hidrocoloides, apósitos de foam, taloneras, aros de goma No posee: no posee ningún grupo de elementos para prevenir UPP

Adultos: sala general - UTI-UCO, guardia- domiciliaria

Pediatría: sala general - área cerrada

Neonatología: área cerrada

Neonatología: menos 28 semanas; 28 a 33 semanas; 34 a 38 semanas; 38 semanas a postérmino

Pediatría: 31 días a 1 año; 1 a 6 años; 7 a 10 años; más de 10 años hasta 17 Adultos: 18 a 40 años; 41 a 60 años; 61 a 75 años; más de 75 años 
Advisory Panel (EPUAP) ${ }^{13}$ para prevalencia, los utilizados en estudios de prevalencia realizados en España por el GNEAUPP' ${ }^{6}$ (2012) y el Conjunto Mínimo Básico de Datos en registros de Úlceras por Presión del GNEAUPP ${ }^{14}$.

En la tabla 1 se enuncian las variables que se utilizaron en el presente estudio ${ }^{15}$

Se calculó la prevalencia bruta (pacientes con UPP/total de pacientes) y la prevalencia de úlceras nosocomiales (pacientes lesionados durante la estancia hospitalaria/total de pacientes lesionados), la localización y la gravedad de las UPP y los elementos de prevención utilizados. Se analizaron también el servicio de internación y las características demográficas de los pacientes.

La información fue recogida por personal de enfermería voluntario de cada institución. Se comenzó con una campaña de difusión con un vídeo explicando en qué consistía el estudio e invitando a quienes se quisieran sumar como investigadores a inscribirse como tales. Se maximizó el alcance del estudio difundiendo en redes sociales y diferentes grupos de whatsApp.

Teniendo en cuenta la complejidad y el alcance del estudio, se diseñó un plan de trabajo que constaba de tres fases: inscripción de voluntarios, recepción de datos, armado de la base de datos y análisis de los datos, y enunciado de conclusiones.

En la primera etapa $(1 / 11 / 17$ al $31 / 5 / 18)$ se recabó información sobre cada investigador, que posteriormente fue agregado al grupo de whatsApp creado para el estudio (siempre que hubiera prestado su consentimiento para ello).

En la segunda etapa (si bien originalmente el periodo de recogida de datos fue propuesto para los meses de junio y julio de 2018, la fecha fue porrogada hasta el 15 de agosto del 2018) se fueron recibiendo los datos enviados por cada investigador y se fue armando la base de datos que posteriormente se usó para el análisis.

Mediante un sistema de claves con resolución de colisiones se aseguró el anonimato de los datos, de forma tal que sin contar con la base de claves no es posible relacionar investigadores con instituciones ni datos.

Para la implantación y ejecución del estudio se tuvo en cuenta la practicidad, velocidad de carga, envío de datos y en el ahorro de insumos como el papel, por lo que la recogida de datos se estructuró sobre dos pilares informáticos que fueron el nexo entre investigadores y analistas de datos.

El primer pilar fue una página web. Esta se diseñó para contener toda la documentación pertinente. De esta manera, se logró accesibilidad en tiempo real y se evitó que cada documento involucrado implicase un interminable intercambio de correos electrónicos, con el consecuente retraso y pérdida de información.

Toda la documentación del estudio, instructivos y acceso a la carga de datos estuvieron concentrados en esta página, dando acceso permanente a los investigadores al contenido que pudiesen necesitar. La web daba acceso a 47 documentos (protocolos, guía práctica, guía de contabilización de datos, consentimientos informados, etc.).

Para poder tener una comunicación más fluida, cada investigador registrado fue incluido en un grupo de whatsApp creado para el estudio. Este medio fue utilizado para comunicar novedades, así como para resolver dudas.

El segundo pilar fueron los formularios de carga online. A través de ellos, los investigadores pudieron cargar los datos en tiempo real. Así, mediante un teléfono inteligente o una tableta y con una conexión a internet, pudieron cargar los datos a medida que los fueron observando, y enviarlos al instante para comenzar con un paciente nuevo. Con ello se evitaban papeles y tener luego que cargar los datos, lo que, "a priori", implicaba perder tiempo y un doble esfuerzo.
Para la tercera etapa (16/8/18 al 28/10/18), los datos de todos los cuestionarios fueron recibidos e incorporados a una base de datos donde se revisaron y se compactó toda la información.

Para el análisis de los datos se utilizó el paquete MS Office 2016 y se emplearon estadísticos de tipo descriptivo.

Con respecto a los principios éticos, el CEECUPPYH-FAE y los demás miembros del equipo interviniente conocían y se comprometieron expresamente a cumplir con los principios éticos de la Declaración de Helsinki, sus modificaciones y las Guías de Buenas Prácticas Clínicas. En el anexo 1 se encuentra una copia del consentimiento informado entregado al paciente.

\section{У RESULTADOS}

Finalizada la $3 .^{\text {a }}$ etapa del estudio, se recibieron 3.785 cuestionarios. Tras la tabulación de datos, se descartaron duplicados y errores, procesándose un total de 3775 cuestionarios.

Participaron 22 de las 24 provincias de Argentina con un total 200 investigadores voluntarios que relevaron datos. Once meses de trabajo.

\section{Características de las instituciones de salud}

Las instituciones de salud representadas en el presente estudio ascienden a 97 , de las cuales 69 son públicas y 28 son privadas.

Cuando se consultó sobre la existencia de algún referente/comité/grupo sobre cuidados de la piel, se constató que en el 30\% de las instituciones existían. En relación con los protocolos, en el 36\% de las instituciones refieren que hay en existencia y en el 60\% se realizó algún tipo de capacitación sobre prevención y tratamiento de UPP. En relación con la realización de estudios previos de prevalencia de UPP, solo el 20\% de las instituciones había realizado alguno y los participantes refirieron que solo fueron sectoriales o internos (UTI; Clínica Médica).

\section{Características de los pacientes (tabla 2)}

\section{Adultos}

Del total de 3755 pacientes contabilizados, 3131 (83,38\%) pacientes son adultos; de ellos, el 57\% está jubilado o en edad de jubilarse (mayores de 60 ańos).

Para representar las edades con mayor frecuencia se utilizó la clase modal, ya que no se tomaron las edades puntuales, pero se dividieron en intervalos de clase; esta representa el 32,64\% de N (61 a 75 años).

En relación con la localización de la unidad de internación que se encontraron en el momento del estudio tenemos:

Internación general: incluye traumatología, cirugía, clínica médica, urología, neurología, neurocirugía, geriatría e internación domiciliaria; está representada por 2175 pacientes (69,47\% de los adultos).

Unidad de Terapia Intensiva-Unidad Coronaria: están representadas por 790 pacientes $(25,23 \%)$.

Internación domiciliaria: pacientes que recibe cuidados en su casa realizados por profesionales; representan 117 pacientes (el 3,74\%).

Guardia/Urgencias: pacientes que se encuentran más de 24 horas internados y que debido a una cuestión de falta de camas para internar pertenecen allí); representan 49 pacientes (1,56\%).

Con respecto al riesgo de padecer UPP, todos los pacientes fueron evaluados por una escala validada, la escala de Braden, puntuándose a 1630 pacientes $(72,50 \%)$ dentro del rango de riesgo moderado y alto. 
Tabla 2. Características de los pacientes

\begin{tabular}{|c|c|c|c|c|c|}
\hline & Edad & $\begin{array}{l}\text { Sector } \\
\text { Interna- } \\
\text { ción }\end{array}$ & $\begin{array}{l}\text { Riesgo } \\
\text { UPP }\end{array}$ & Género & $\mathbf{N}$ \\
\hline \multirow{4}{*}{$\begin{array}{l}\text { 올 } \\
\text { 흔 }\end{array}$} & $\begin{array}{l}18 \text { a } 40 \text { años } \\
\quad 601\end{array}$ & $\begin{array}{l}\text { Sala } \\
\text { General } \\
2175\end{array}$ & $\begin{array}{c}\text { Riesgo } \\
\text { bajo } \\
1501\end{array}$ & $\begin{array}{c}\text { Femenino } \\
1597\end{array}$ & \multirow{4}{*}{3131} \\
\hline & $\begin{array}{l}41 \text { a } 60 \text { años } \\
754\end{array}$ & $\begin{array}{l}\text { UTI-UCO } \\
790\end{array}$ & \multirow{2}{*}{$\begin{array}{c}\text { Riesgo } \\
\text { medio } \\
769\end{array}$} & \multirow{3}{*}{$\begin{array}{l}\text { Masculino } \\
1534\end{array}$} & \\
\hline & $\begin{array}{l}61 \text { a } 75 \text { años } \\
1022\end{array}$ & $\begin{array}{l}\text { Guardia } \\
\quad 49\end{array}$ & & & \\
\hline & $\begin{array}{c}\text { Más de } 75 \text { años } \\
754\end{array}$ & $\begin{array}{l}\text { I. Domici- } \\
\text { liaria } \\
117\end{array}$ & $\begin{array}{l}\text { Riesgo } \\
\text { alto } \\
861\end{array}$ & & \\
\hline \multirow{4}{*}{ 空 } & $\begin{array}{c}31 \text { días a } 1 \text { año } \\
382\end{array}$ & \multirow{2}{*}{$\begin{array}{c}\text { Área } \\
\text { cerrada } \\
102\end{array}$} & $\begin{array}{c}\text { Riesgo } \\
\text { bajo } \\
285\end{array}$ & \multirow{2}{*}{$\begin{array}{l}\text { Femenino } \\
207\end{array}$} & \multirow{4}{*}{431} \\
\hline & $\begin{array}{c}1 \text { a } 6 \text { años } \\
136\end{array}$ & & \multirow{3}{*}{$\begin{array}{c}\text { Riesgo } \\
\text { medio } \\
74 \\
\text { Riesgo } \\
\text { bajo } \\
72\end{array}$} & & \\
\hline & 7 a 10 años & \multirow{2}{*}{$\begin{array}{c}\text { Sala } \\
\text { General } \\
329\end{array}$} & & \multirow[b]{2}{*}{$\begin{array}{l}\text { Masculino } \\
224\end{array}$} & \\
\hline & $\begin{array}{l}\text { Mas de } 10 \text { años } \\
\quad 61\end{array}$ & & & & \\
\hline \multirow{4}{*}{$\begin{array}{l}\text { 뜸 } \\
\text { 응 } \\
\text { 흠 } \\
\text { 인 }\end{array}$} & $\begin{array}{c}\text { Menos de } 28 \text { semanas } \\
14\end{array}$ & \multirow{4}{*}{$\begin{array}{c}\text { Área } \\
\text { cerrada } \\
193\end{array}$} & \multirow{2}{*}{$\begin{array}{l}\text { Con riesgo } \\
118\end{array}$} & \multirow{2}{*}{$\begin{array}{c}\text { Femenino } \\
107\end{array}$} & \multirow{4}{*}{193} \\
\hline & $\begin{array}{c}\text { De } 28 \text { a } 33 \text { semanas } \\
46\end{array}$ & & & & \\
\hline & $\begin{array}{c}\text { De } 34 \text { a } 38 \text { semanas } \\
85\end{array}$ & & \multirow{2}{*}{$\begin{array}{c}\text { Sin riesgo } \\
75\end{array}$} & \multirow{2}{*}{$\begin{array}{l}\text { Masculino } \\
86\end{array}$} & \\
\hline & $\begin{array}{c}\text { De } 38 \text { semanas o más } \\
48\end{array}$ & & & & \\
\hline
\end{tabular}

\section{Pediatría}

Del total de la población contabilizada: 431 pacientes pertenecen a la categoría de pediatría $(11,48 \%)$; la mayor frecuencia de los pacientes se encuentra en el rango de edad de entre 31 días y 1 ańo.

En relación con la unidad de internación pediátrica: 329 pacientes $(76,33 \%)$ estaban en unidades de internación general pediátrica y 102 $(23,67 \%)$, en área crítica o intensiva (según la denomine la institución).

\section{Neonatología}

Los 193 pacientes neonatos representan el 5,14\% de la muestra, y la mayor representación se encuentra dentro del intervalo de 34 a 38 semanas de gestación (85 pacientes) con el 44,04\%.

\section{Prevalencia de UPP}

De los pacientes adultos contabilizados en el momento del estudio (3131 pacientes), 706 presentaron UPP. Esto representa una prevalencia bruta del 22,55\%.

Si consideramos a las UPP producidas en el Servicio (nosocomiales), el porcentaje de pacientes con al menos una UPP que se ha producido desde su ingreso es del 48,30\% de los pacientes.

De los pacientes pediátricos en el momento del estudio (431 pacientes), 36 presentaron UPP; esto representa una prevalencia bruta del 8,35\%.

Si consideramos las UPP producidas en el Servicio, el porcentaje de pacientes prevalentes con UPP nosocomiales asciende al 69,44\%.

De los pacientes de Neonatología en el momento del estudio (193 pacientes), 8 presentaron UPP; esto representa una prevalencia bruta del $4,15 \%$. Cabe destacar que todas las lesiones que se registraron fueron producidas en el Servicio.

\section{Características de las UPP}

En la figura 1 se puede observar, de forma comparativa entre la población adulta $(\mathrm{N}=3131)$, la ubicación anatómica donde se produjeron las lesiones.

En la figura 2 se expone la clasificación de las UPP según su gravedad.

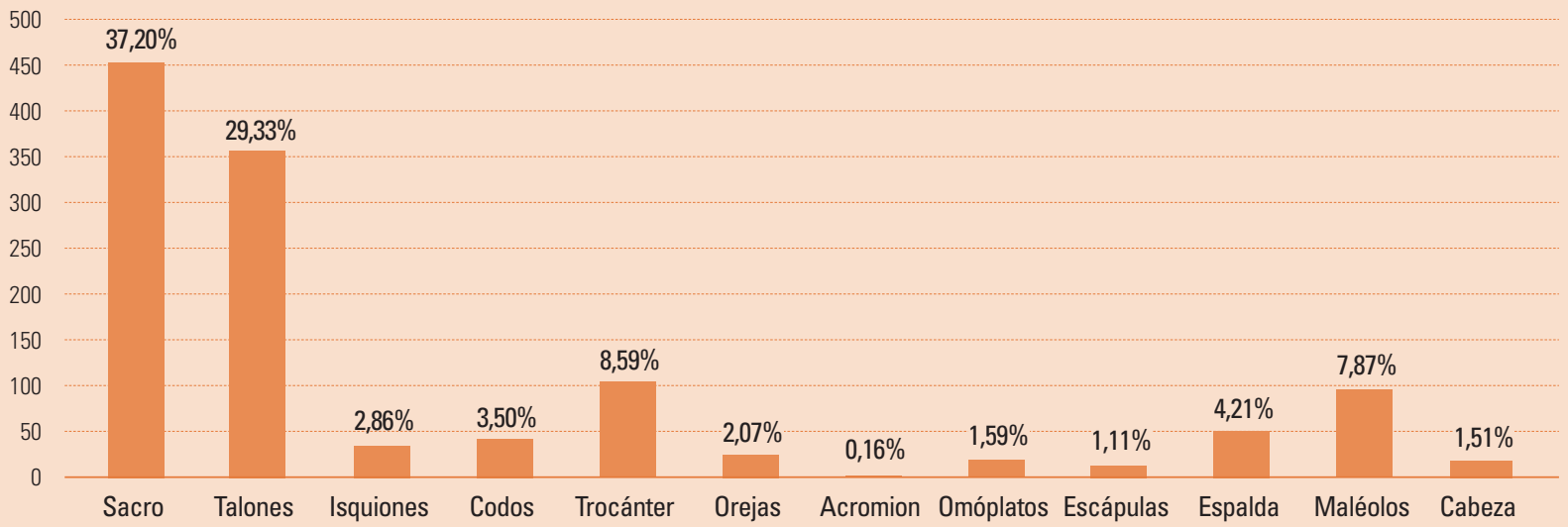

Figura 1. Distribución anatómica de las UPP en población adulta. 
En la figura 3 se muestra, de forma comparativa entre la población pediátrica $(\mathrm{N}=431)$, la ubicación anatómica donde se produjeron las lesiones.

En la figura 4 se puede observar la clasificación de las UPP según su gravedad.

En la figura 5 se detalla, de forma comparativa entre la población de neonatos $(\mathrm{N}=193)$, la ubicación anatómica donde se produjeron las lesiones.

En la figura 6 se puede observar la clasificación de las UPP según su gravedad.

\section{Recursos utilizados en la prevención de UPP}

El $60 \%$ de los pacientes no posee ningún tipo de elementos para prevención; el 23\% posee al menos 1 grupo de elementos de prevención; el $15 \%$ utiliza 2 grupos de prevención, y solo el 2\% de los pacientes utiliza 3 grupos de prevención.

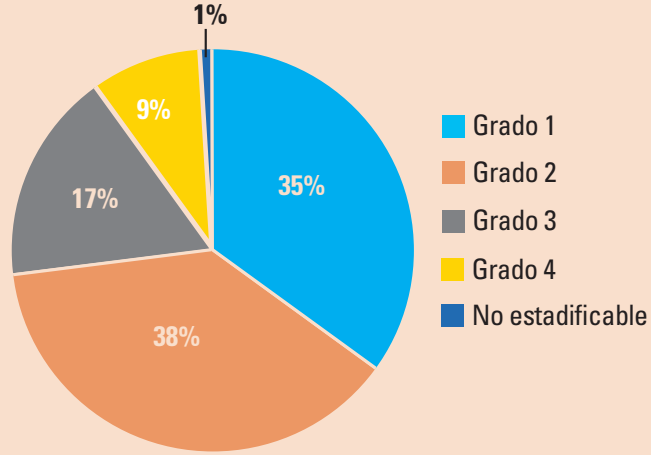

Figura 2. Clasificación de las UPP según gravedad en población adulta.

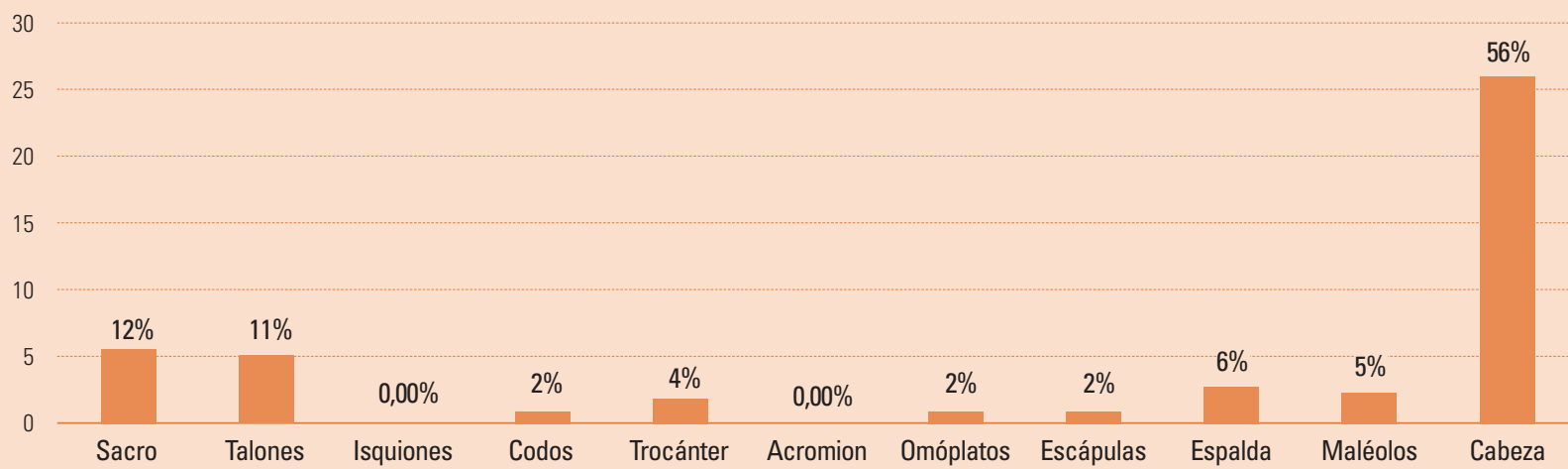

Figura 3. Distribución anatómica de las UPP en población pediátrica.

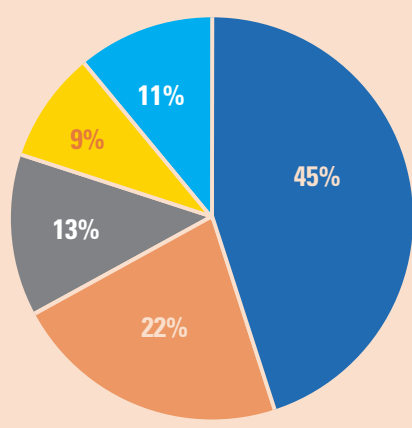

Grado 2 No estadificable

Grado 3

Figura 4. Clasificación de las UPP según gravedad en población pediátrica.

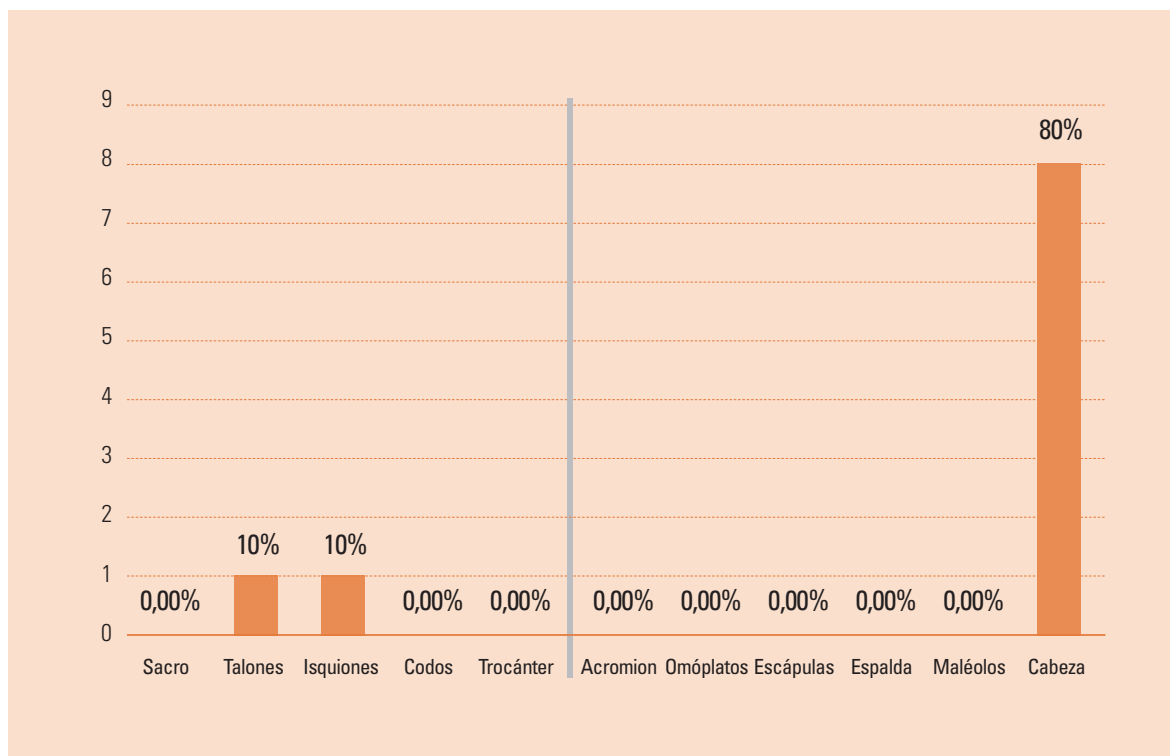

Figura 5. Distribución anatómica de las UPP en población de neonatos. 


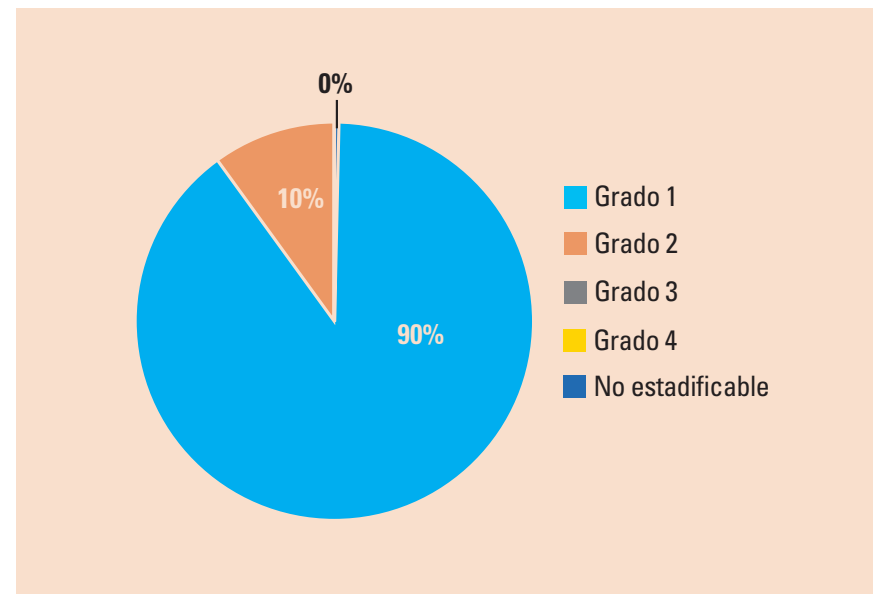

Figura 6. Clasificación según la gravedad en población de neonatos.

\section{У DISCUSIÓN}

De la recolección y procesamiento de los datos surge que si bien en el $31 \%$ de las instituciones funcionan comités relacionados con la temática, el 36\% posee protocolos de actuación, el 40\% valora el riesgo de padecer UPP con una escala validada y más de la mitad ha recibido capacitación, llama la atención que nos encontremos con una prevalencia bruta tan elevada en comparación con otros países limítrofes, y sobre todo la elevada prevalencia de UPP nosocomiales, que asciende al $50 \%$ del valor bruto.

Esta estadística no queda reflejada en la comunicación bidireccional aportada (WhatsApp) como vía de enlace para enfermeros/as del país que aportaron datos, ya que por medio de este canal se podían hacer consultas sobre el estudio en sí mismo o sobre algún caso clínico. Por un lado, quedó al descubierto la deficiencia en capacitación sobre prevención y tratamiento basada en la mejor evidencia científica, y por otro, si bien hay instituciones que poseen protocolos, y utilizan escalas de valoración de riesgo de padecer UPP, es necesario que estas revisen las estrategias de implementación y cumplimiento.

A diferencia de otros estudios realizados sobre el mismo tema, en nuestro sistema de salud figuran administrativamente varios niveles de atención; el segundo nivel de atención (diagnóstico y tratamiento) es el que históricamente tiene un papel principal junto al nivel de gravedad en relación con los cuidados y tratamiento. Es por eso por lo que dentro de la variable "lugar de internación" se categorizó de la siguiente manera: para cuidados generales de cualquier especialidad: sala general: incluye clínica médica y otras especialidades quirúrgicas; para cuidados intensivos: UTI-UCO; internación domiciliaria: pacientes que se encuentran en su domicilio, pero con asistencia médica y de enfermería. Si bien esta variable será difícil de extrapolar en estudios que se realicen en otros países, incluso será difícil de comparar, esta es la mejor manera que encontramos para que los datos sean realmente útiles para nuestro país.

Si nos detenemos en los resultados referidos al lugar anatómico de las lesiones en adultos, se puede observar que se presentan en talones y sacro, seguido por trocánteres y maléolos. Resultados similares se han encontrado en otros estudios similares; esto es un signo de que las medidas de prevención con respecto a la rotación de pacientes no está naturalizado dentro del personal de enfermería en nuestro país; con respecto a la población pediátrica, las lesiones se encuentran en un porcentaje descendiente desde la cabeza, seguido por el sacro; esto nos indica que los profesionales deberán hacer hincapié en la prevención de lesiones por dispositivos médicos, como así también en la rotación de posición en los pacientes; con respecto a la población de neonatos, si bien las lesiones se presentan con menor gravedad, en las orejas son las de mayor frecuencia; hecho que se repite como en la población pediátrica.

Finalmente, en relación con la variable "insumos y/o elementos utilizados para prevención”, cabe destacar que, a diferencia de otros países, en Argentina ninguna obra social, seguro médico privado o público cubre los costos de los mismos; en consecuencia, los gastos corren a cargo de cada institución donde el paciente esté internado. Partiendo de esta realidad, vemos que, dentro de esta variable, la categoría "no posee ninguno" lamentablemente es la de mayor frecuencia, seguida por la categoría "SEMP". Es necesario aclarar que, en nuestro país, la comercialización del sobrecolchón que en España denominan "huevera” es la más accesible en el mercado, ya que las que aconseja la evidencia científica son mucho más costosas y de difícil acceso. Este tipo de sobrecolchón es el que adquieren instituciones públicas y privadas, incluso son las que proveen algunas medicinas prepagas y seguros médicos cuando el paciente posee internación domiciliaria.

Le siguen en orden de importancia la utilización de insumos de protección local como ácidos grasos hiperoxigenados (AGHO), apósitos de hidrocoloide y aceites esenciales; todos estos son costeados de forma particular por los pacientes o sus familiares.

Cabe destacar que todos los resultados obtenidos de la población pediátrica, así como la neonatología deben tomarse con la precaución necesaria, ya que no se puede garantizar su representatividad a nivel nacional, puesto que el número de pacientes relevados es mucho menor (suponemos que esto es debido a que las instituciones que cuidan a estos pacientes aluden "defender" los derechos del niño y quieren evitar posibles conflictos legales con los tutores). No obstante, y debido a que los resultados obtenidos son estadísticamente consistentes con resultados a nivel nacional, se los puede considerar orientativos.

\section{Agradecimientos}

Queremos agradecer la colaboración del Dr. Joan-Enric Torra i Bou (SAPIENS Consultants, www.sapiens-fhwc.com) en la revisión y aportaciones para la redacción del manuscrito del presente artículo.

Los coordinadores del estudio quieren manifestar un agradecimiento especial a todos los profesionales de enfermería argentinos que colaboraron en "La Maratón Nacional": sin ellos no hubiera sido posible:

Carla Gimena Salomóne, Romina D'Aranno, Nancy Aguirre, Norberto Bandeo, Verónica Andrea Cahullan, Ramona Elizabet Monje, Rita Pérez, Marina Patricia Funes, Alicia Darchez, Viviana Acuña, Claudia Paola Ayamilla, Héctor Omar Díaz, Débora Daiana Escobar, Roxana Vallejos, Milca Cecilia Medina, Luciana Ortiz Olmedo, Verónica Ramírez, Orlando Galarza, David Pini, Romina Chumba, Diego Gómez, Miriam Susana Luna, Marcela Carina Viveros, Santos Segovia, Mariana Elizabeth Vilte, Mario José Alberto Lobos, Laura Raquel Deydar, Delia Mansilla, Marcos Ezequiel Garay, Graciela Rosello, Ofelia Elizabeth Pérez, Gabriela Cano, Sergio Óscar Orellana, Marianela Vanesa Juárez, Miguel Ángel López, Paula Carrizo, Virginia Lourdes Vargas, Roberto Olivarez, Mirtha E. Galarza de la Rosa, Miguel Ángel Galarza, María Laura Acuña Estela del Valle Rodrijuez, Erika Quisbert, Marcelo Macchi, Judith Medina, Melina Gabriela Rivas, Sergio Castañeira, Alicia Claudia Sposito, Antonella Granados, Laura Cazuza, Claudia Castillo, María Nahuelquir, Daniela Fernanda Gallegos, Adriana Armendariz, Hernan Aníbal Lascano, Cristian Arroyo, Rosalía Paz, Nora Brizuela, Graciela Verónica Burgos, María Silvia Vélez, Walter Jiménez, Gladys Alicia Toranzo, María del Carmen Cáceres, Antonia María Moreno, Patricia Alejandra Delgado, Irma Viviana Lucas, Cristian Enríquez, María Angélica Ibarra, Franco M. Oscar Rojas, José María Benítez, Griselda Escobar, Florencia Caballero, Víctor Orlando González, Fátima Ruiz, Sonia Alfonso, Claudia Moreno, Verónica Manfrin, Victoria Giraldo, Alejandro Gómez Poblete, Leticia Sánchez, Laura Paredes, Andrea Rial, Carlos Alejandro Bascary, Alicia Mena, Nicolás Martín Schuster, Sivia Soledad Leiva, Carolina Sobrero, 
Carina Sánchez, Ariel Rivas, María Jimena Alarcón, Jésica Wurm, María F. Domínguez, Gabriela Alarcón, Ercilio Miguel Martínez, José Luis Ávalos, Gabriela Alarcón, Florentín Dario Martínez, Carina Limbozzi, Claudia Green, Arnaldo Romero, Cristina Enríquez,Telma Mirian Zarate, Lidia Marcela Martínez, Dora Gómez, Luis Horacio Moreno, Dina Esther Ifran, Verónica Cortese, Sandra Bayser, Walter Alejandro Journet, María Josefina Soto, Marcela Verónica Arancibia, Ángela Patricia Díaz

\section{Aspectos éticos}

Se cumplieron con todos los ítems de las normas éticas y la Declaración de Helsinki. Se asignó a cada investigador/colaborador un código desvinculando la identificación real del centro donde se relevaron los datos. Se preparó una hoja de información a pacientes y un modelo de consentimiento informado a quienes lo solicitaron.

Conflicto de intereses

Los autores del Estudio no poseen ningún conflicto de intereses. El estudio no recibió ningún tipo de financiación.

\section{Anexo I. Consentimiento Informado para pacientes que participaron del estudio Información sobre el estudio de investigación sobre prevalencia nacional de UPP}

\section{1. ¿Cuál es el objetivo del estudio?}

El propósito del estudio es investigar a nivel nacional la prevalencia de úlceras por presión en pacientes internados en instituciones de salud.

\section{2. ¿Quién puede participar en este estudio?}

Cualquier persona que esté internada en una institución de salud; en el caso de ser menor de edad, sus padres deberán prestar la conformidad.

\section{3. ¿Por qué debería considerar mi participación como sujeto de investigación en este estudio?}

Para contribuir al desarrollo del conocimiento sobre las características de los pacientes internados, para poder proponer mejoras en la calidad de atención de los pacientes.

4. ¿Tengo necesariamente que participar en este estudio? ¿Si acepto participar, puedo cambiar de opinión o retirarme? No tiene necesariamente que participar en el estudio. Si participa y cambia de opinión, puede retirarse.

5. ¿Si decido participar en el estudio, en qué consisten precisamente las evaluaciones, y qué tipo de tratamientos o procedimientos me van a practicar?

Se le realizará una inspección visual y se valorará la integridad cutánea de todo su cuerpo.

6. ¿Qué peligros podría experimentar en este estudio, y qué harán los investigadores para reducir el riesgo de que estos se presenten? Su participación en el estudio no reviste ningún peligro.

7. ¿Qué harán los investigadores para asegurar que la información que recolectarán sobre mí no caerá en manos equivocadas? Usaremos un código en vez de su nombre. No publicaremos ningún dato personal suyo, solo datos grupales.

8. ¿Qué beneficios personales puedo yo esperar al participar en este estudio? No tendrá ningún beneficio salvo la contribución al conocimiento sobre su propia patología.

\section{9. ¿En qué podría este estudio beneficiar a otros?}

Otros pacientes se podrían beneficiar si se toman futuras decisiones sobre prevención y tratamiento de úlceras por presión en función de los datos aportados por el estudio.

10. ¿Qué harán los investigadores si sufro algún daño durante el estudio?

No sufrirá ningún daño durante el estudio.

11. ¿Recibiré algún pago por participar en este estudio? No habrá pagos por participar en este estudio.

12. ¿Una vez que yo haya ingresado como sujeto de estudio, ¿a quién tendría que dirigirme para averiguar más acerca del estudio o para hacer llegar algún reclamo respecto al trato que hubiese recibido?

Deberá dirigirse al CEECUPPYH de la Federación Argentina de Enfermería.

13. ¿Si decido no participar en este estudio, ¿qué me puede suceder, o qué otras opciones tengo si necesito tratamiento? Nada puede sucederle si no participa en el estudio. Si necesita tratamiento lo puede hacer a su elección, nosotros solo vamos a evaluar y aconsejarle. 


\section{Documentación del consentimiento}

\section{Después de que firme el documento, ¿quien lo guardará?}

El documento de consentimiento será archivado en el Departamento tal y será guardado por el Departamento de Enfermería.

del Hospi-

\section{Consentimiento informado}

Estoy dispuesto a participar en el proyecto de investigación sobre Prevalencia de UPP en Internación 2018. Participaré como informante respondiendo a todas las preguntas que me hagan sin esperar pago o retribución. Mi intención es cooperar en el desarrollo de conocimiento útil en relación con el problema de las UPP.

He leído la información descrita y mis preguntas acerca del estudio han sido respondidas satisfactoriamente. Al firmar esta copia, indico que tengo un entendimiento claro del proyecto.

Firma

Al sujeto de investigación he entregado información sobre el estudio, y en mi opinión esta información es precisa y suficiente para que el sujeto entienda completamente la naturaleza, los riesgos y beneficios del estudio, y los derechos que tiene en tanto sujeto de investigación. No ha existido coerción ni ha actuado bajo influencia alguna. He sido testigo de que el sujeto firmó el documento.

Nombre del investigador

Firma del investigador

Ciudad - País 


\section{У BIBLIOGRAFÍA}

1. Grupo Nacional para el Estudio y Asesoramiento en Úlceras por Presión y Heridas Crónicas (GNEAUPP). Mesa de debate: las úlceras por presión, un reto para el sistema de salud y la sociedad "Repercusiones a nivel epidemiológico, ético económico y legal. Logroño, 2003.

2. Soldevilla J, Verdú J, Torra J. Impacto social y económico de las úlceras por presión. Gerokomos. 2007;18(4):201-10.

3. Allman RM. The impact of pressure ulcers on health care cost mortality. Adv Wound Care. 1998;11(3 suppl):2.

4. Slawomirski L, Auraaen A, Klazinga N. "The economics of patient safety: Strengthening a value-based approach to reducing patient harm at national level", OECD Health Working Papers, №. 96. Paris, OECD Publ.; 2017. https://doi.org/10.1787/5a9858cd-en

5. Hauck K, Wang S, Vincent C, Smith PD. Healthy life years lost and excess bed-days due to 6 patient safety incidents empirical evidence from English Hospitals. Med Care 2017-55(2):125-30.

6. Pancorbo-Hidalgo PL, García-Fernández FP, Torra i Bou J-E, Verdú
Soriano J, Soldevilla-Agreda JJ. Epidemiología de las úlceras por presión en España en 2013: 4. Estudio Nacional de Prevalencia. Gerokomos. 2014;25(4):162-70

7. Clark M. Bours Defloor. Pressure Ulcer prevalence monitoring Project. Summary report on the prevalence of pressure ulcers EUPAP Review 2002. EPUAP, 2014

8. Palacios JL. Normas sobre la seguridad del paciente y calidad de la atención de prevención úlceras o lesiones por presión (UPP) en pacientes hospitalizados. Disertación Congreso Nacional Seguridad del Paciente. Buenos Aires; FAE; 2014.

9. García FP, Pancorbo HP, Soldevilla JJ, Blasco GC. Escalas de valoración del riesgo de desarrollar úlceras por presión. Gerokomos. 2008;19(3):136-44.

10. García-Fernández FP, Pancorbo-Hidalgo PL, Soldevilla Agreda JJ. Escalas de valoración del riesgo de desarrollar úlceras por presión en la infancia. Gerokomos. 2011;22(1):26-34.
11. Curley MA, Razmus IS. "Predicting pressure ulcer risk in pediatric patients: the Braden 0 Scale", Nursing Res. 2003;52(1):22-3.

12. Andrades P, Sepúlveda S, González J. Curación avanzada de heridas. Rev. Chilena de Cirugía. 2004;56(4):396-403.

13. Prevención y tratamiento de las Úlceras por Presión: Guía de consulta rápida. En: Haesler E, ed. Perth, Australia; Cambridge Media. Versión Española por GNEAUPP.

14. Ibars-Moncasi P, San Sebastián Domínguez JA, Soldevilla-Agreda JJ Conjunto Mínimo Básico de Datos en registros de Úllceras por Presión (CMBD-UPP. Serie de documentos GNEAUPP N ํ 12. Logroño, 2012

15. García MR. Trabajo de Fin de Máster. Proyecto de Investigación. Prevalencia de UPP en pacientes ingresados en Instituciones de salud residencias geriátricas de Uruguay. Universidad de Cantabria; 2013. 